\title{
EUROPA
} \section{Zwischen Markt und Mildtätigkeit}

\author{
Udo Krolzik
}

Die Sozialwirtschaft ist nicht nur von Europa betroffen, sie kann Europa mitgestalten. Am Beispiel der Diakonie und ihrer Angebote für die Altenpflege können Chancen, Strategien, Perspektiven gezeigt werden.

Die Formulierung »zwischen Markt und Mildtätigkeit « kann im Sinne eines Entweder-oder verstanden werden und würde dann bedeuten, dass Diakonie sich zu entscheiden hat - für Markt oder für Mildtätigkeit. In der Tat wird gelegentlich die Situation der Diakonie in der europäischen Entwicklung von manchen Vertretern der Diakonie - insbesondere von Verbandsvertretern - so verstanden. Diese Sicht ist dann häufig verbunden mit dem Vorwurf oder zumindest dem Verdacht, dass die unternehmerische Diakonie im Sinne einer solchen Entweder-oder-Entscheidung dazu neige, sich ganz für den Markt zu entscheiden.

Eine andere Verhältnisbestimmung könnte zu der Auffassung gelangen, dass Diakonie in der Mitte stecke - zwischen Markt und Mildtätigkeit - wie zwischen Stamm und Borke, also in einer unglücklichen Position. Wenn Vertreter der unternehmerischen Diakonie ihre Situation so einschätzen und ihre Arbeit in dieser unglücklichen Position sehen, dann wird häufig dieser Weder-Fischnoch-Fleisch-Zustand beklagt als Verlust der Mildtätigkeit unter den Zwängen des Marktes.

Ich verstehe das Thema »Zwischen Markt und Mildtätigkeit « unter der Herausforderung »Europa sozial gestalten «so, dass Diakonie das ganze Spektrum von marktgängigen bis zu spendenfinanzierten Leistungen umfasst, dies als Chance für eine diakonische Gestaltung Europas erkennt und zu deren Realisierung Strategien und Perspektiven entwickelt. Von daher werde ich versuchen, auf folgende drei Fragen zu antworten:

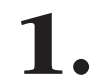
Welche Chancen bietet dieses Spektrum der Diakonie für ein diakonisches Gestalten Europas? Um dies zu konkretisieren, wähle ich die Angebote der Altenarbeit.

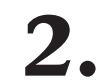

Welche Strategien lassen sich in der diakoni- schen Altenarbeit zur Realisierung dieser Chancen entwickeln? 3. Welche Perspektiven bietet Europa für die diako-
nische Altenarbeit?

Ich beginne mit einer kleinen Anekdote: Die Industrieund Handelskammer (IHK) in Bayern informierte Kleinunternehmer über grenzüberschreitende Förderung in Europa. Anschließend fragte ein Unternehmer, ob diese Förderung auch gelte, wenn er ein Unternehmen in Württemberg errichten wolle.

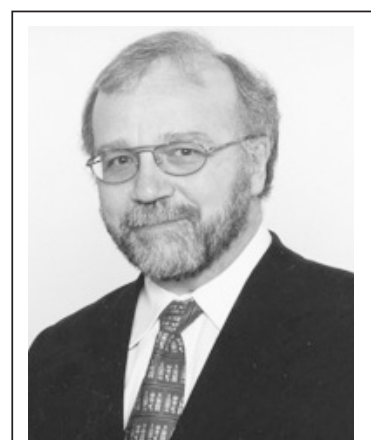

Dr. Udo Krolzik (58) ist Vorsitzender des Vorstandes des Evangelischen Johanneswerkes e. $V$. in Bielefeld. Er hat Betriebswirtschaft und Theologie in Hamburg und Edinburgh studiert und war wissenschaftlicher Assistent am kirchen- und dogmengeschichtlichen Seminar der Universität Hamburg. Von 1989 bis 1993 war er Pastor in Hamburg-Hummelsbüttel, dann wurde er theologisches Mitglied im Vorstand des Evangelischen Johanneswerkes, dessen Vorsitz er 1996 übernahm. E-Mail udo-krolzik@johanneswerk.de

Ein solches Denken ist kein Spezifikum Bayerns. Solche Grenzen sind auch in den Köpfen in Westfalen bezogen auf das Rheinland, in Baden bezogen auf Württemberg und jeweils umgekehrt. Und nicht nur Kleinunternehmer denken so kleinräumig, sondern auch diakonische Träger. Wenn wir nicht einmal Nordrhein-Westfalen oder Baden-Württemberg diakonisch gemeinsam gestalten können, wie wollen wir dann Europa gemeinsam diakonisch gestalten?

Es könnte allerdings sein, dass gerade die europäische Herausforderung, grenzüberschreitend diakonisch zu gestalten und zu wirken, auch unsere deutschen Landesgrenzen weit zurücktreten lässt.

\section{Chancen}

Es gilt zunächst den europäischen Markt für Altenarbeit genauer zu beschreiben.

- Wir haben einen wachsenden Markt.

- Demografische Entwicklung: Es gibt in ganz Europa zwei demografische Trends, die enorme Auswirkungen auf die Sozialdienste der Länder haben, nämlich die immer geringere Geburtenrate und der Trend zu einem immer höheren Lebensalter. Der erste Trend ist wichtig, da er die künftige Verfügbarkeit erwachsener Kinder beeinflusst, die ihre abhängigen älteren Eltern pflegen. (1) Der zweite, weil die Wahrscheinlichkeit der Pflegebedürftigkeit mit dem Alter deutlich an- 
steigt und der Hauptanteil schwerpflegebedürftiger Personen in Heimen versorgt wird. Im Europa weist nach den Zahlen aus dem Jahre 2003 (2) bei den 25 Mitgliedsstaaten der Europäischen Union Deutschland die niedrigste Geburtenrate auf mit 8,6 Geburten pro 1.000 Einwohnern, dann Griechenland mit 9,3 und Italien mit 9,4 bei den alten EU-Ländern.

- Gesellschaftliche Entwicklung: Die Zahl der Ein-Personen-Haushalte wächst in den europäischen Ländern. Dies hat natürlich Auswirkungen auf die zukünftigen Pflegeerfordernisse. Dabei bilden sich neue Netze aus Verwandten, Freunden und Nachbarn, die Teile der Betreuung übernehmen werden. Ein zusätzlicher Faktor ist der Trend, dass mehr Frauen - die traditionellen Betreuerinnen der Familie - in den Arbeitsmarkt eintreten und daher weniger verfügbar für die Pflege der abhängigen Familienmitglieder sind. Das traditionelle Familienmodell ändert sich angesichts der steigenden Zahl der alleinerziehenden Eltern und Familien, die von Scheidung und Wiederheirat betroffen sind. Dies sind komplexe Themen mit großen Folgen für die Zukunft der Betreuung in der Familie und der Sozialdienste.

- Bevölkerungsbewegungen: Die Veränderungen in Mittel- und Osteuropa nach 1989 führten dazu, dass viele Menschen in andere Länder gezogen sind, um politisches Asyl und bessere Beschäftigungsmöglichkeiten zu finden. Daraus folgt eine größere ethnische und kulturelle Vielfalt in den Bevölkerungen vieler europäischer Gesellschaften. Länder, die viele Flüchtlinge und Asylbewerber aufnehmen, müssen kulturell angemessene Sozialdienste zur Verfügung stellen, was Fragen für das Gastland aufwirft. Die Kosten sind nur einer von vielen Faktoren. (3)

Schon seit Jahren sind Menschen aus den klimatisch ungünstigen Regionen in die klimatisch angenehmeren Regionen gezogen, insbesondere im Ruhestand. Teilweise waren diese Wechsel gesundheitlich begründet, teilweise einfach durch die Urlaubserfahrungen und gestiegenen finanziellen Möglichkeiten seit den 1980er Jahren. Diese mittlerweile gealterten Migranten brauchen Unterstützung und Pflege an ihrem neuen Lebensort. Mit ihrem Menschenbild und ihrer Fachlichkeit ist Diakonie mittlerweile nicht nur in anderen europäischen Ländern für die dort lebenden Ausländer vertrauenswürdiger und geschätzter Dienstleistungsanbieter, sondern auch für die dort lebende Bevölkerung.

Von besonderer Bedeutung sind und werden zukünftig insbesondere die jungen Arbeitsmigranten sein, die aufgrund ihrer Arbeitslosigkeit oder des Lohngefälles unter den EU-Staaten emigrieren. Es muss jedoch beachtet werden, dass diese Bevölkerungsbewegungen in der Vergangenheit von Süd- nach Nordeuropa keineswegs so stark wie befürchtet waren. Deutschland und Österreich haben den Zugang zum Arbeitsmarkt weiterhin reguliert. Insofern - so stellte das Bildungswerk Weiterdenken der Heinricht-Böll-Stiftung im April dieses Jahres fest - ist eine Umlenkung vonstatten gegangen, da Menschen, die eigentlich in diese Länder immigriert wären, nach Großbritannien und Irland gegangen sind. Schweden hingegen hatte kaum Zuwanderungen zu verzeichnen. Interessant wird es dann, wenn Deutschland den Arbeitsmarkt freigibt, denn zu vermuten ist, dass die Ballungsräume rund um die Großstädte dies zu spüren bekommen werden.
Die Kombination von Veränderungen in Demografie und Einstellung zur Betreuung in der Familie wird sich stark auf die Nachfrage nach sozialen Dienstleistungen für ältere Menschen im 21. Jahrhundert auswirken. Das Problem, ausreichend bezahlbare Pflege für ältere Bürger zur Verfügung zu stellen, hat vermutlich die größte Priorität für die europäischen Sozialdienste, zusammen mit der Notwendigkeit, von den Erfolgen anderer Länder in diesem Bereich zu lernen.

Gleichzeitig stellen die Wanderungsbewegungen uns vor ganz neue Probleme der interkulturellen Pflege. Die Freizügigkeit der Arbeitnehmer, die in Deutschland für die Beitrittsländer zunächst ausgesetzt ist, verstärkt diese Herausforderung. Diakonie wird dorthin gehen, wo die Menschen sie brauchen.

\section{- Wir haben mehr Markt}

Das hohe Wachstumspotenzial einerseits und die steigende Leistungsbezogenheit der Finanzierungsstruktur nach dem Prinzip »gleiches Geld für gleiche Leistung « andererseits machen den Sozialmarkt auch attraktiv für private Anbieter. Im Rehabilitationsbereich stellen die Privaten im Jahr 2000 knapp 60 Prozent der Einrichtungen, bei ambulanten Pflegediensten sind es mehr als die Hälfte, bei Alteneinrichtungen ein knappes Drittel und bei Akutkrankenhäusern immerhin 20 Prozent mit raschem Anstieg!

Zunehmend treten finanzstarke europäische Mitbewerber auf, die auch über ein Know-how im Dienstleistungsbereich verfügen. Da sie als nichtgemeinnützige Kapitalgesellschaften in der Regel mehr Kapital als gemeinnützige Träger zur Verfügung haben oder sich dieses günstiger beschaffen können, kaufen sie immer mehr und häufig auch konfessionelle Einrichtungen auf.

Hinzu kommt, dass diese privaten Anbieter sich konsequent an ökonomischen Maßstäben ausrichten und größere Freiheiten beim Arbeitsrecht haben, mit der sie in ihren Einrichtungen die Kostenstruktur zu ihren Gunsten verbessern können.

Diese Entwicklung zu mehr Markt ist in Deutschland durch die Pflegeversicherung und insbesondere durch die Reform des Paragrafen 93 des damaligen Bundessozialhilfegesetzes (BSHG) von 1994 bewusst in Gang gesetzt worden. Sie entspricht der inneren Logik der Europäischen Union, die einen Binnenmarkt will und dabei die Dienstleistungen - auch die sozialen Dienstleistungen - nicht aussparen will, wie das europäische Vergaberecht und die Diskussion um die Dienste vom allgemeinen wirtschaftlichen Interesse zeigen.

Die Ausschreibungspraxis in Nordrhein-Westfalen und in einigen neuen Bundesländern hat dazu geführt, dass Unternehmen aus anderen Bereichen oder aus anderen europäischen Ländern sich in der Jugendhilfe beworben haben. Der Landschaftsverband Westfalen Lippe wollte diese Ausschreibungspraxis auch auf das betreute Wohnen in der Behindertenhilfe ausweiten.

Mehr Markt heißt auch mehr europäischer Markt und gerade bei den Personalkosten sind noch erhebliche Unterschiede zu verzeichnen. Die Arbeitnehmerfreizügigkeit steht 
unter Übergangsbestimmungen, die Niederlassungsfreiheit nicht. So können Selbstständige aus den Beitrittsländern sich sehr wohl niederlassen und auch in vernetzten Strukturen arbeiten, Firmen können Schlüsselpersonal mitbringen, in Grenznähe können Mitarbeiter morgens über die Grenze zur Arbeitsstelle gebracht werden und abends wieder zurück, Mitarbeiter können bis zu 18 Monate in Deutschland tätig werden, um sich beruflich oder sprachlich fortzubilden usw. Interessanterweise gibt es auch eine zunehmende West-Ost-Wanderung, wobei es sich aber fast immer um Menschen mit sehr hohen Bildungsabschlüssen handelt, die oft in den Grenzregionen arbeiten.

\section{Strategien}

Auf diesem Hintergrund müssen wir eine Strategie entwickeln, um uns auf einen europäischen Markt einzustellen.

- Europäische Anbieter in Deutschland: Angesichts europäischer Mitbewerber müssen wir unser diakonisches Profil deutlich herausstellen und ebenso die Tatsache, dass wir keine Gewinnabsichten haben. Gleichzeitig werden wir uns angesichts der preisgünstigeren ausländischen Anbieter, der engeren finanziellen Rahmenbedingungen und des wachsenden Bedarfes an Pflegeleistungen neue Ressourcen und veränderte Versorgungsstrukturen erschließen müssen, wenn diakonische Angebote auch weiterhin die individuellen Bedürfnisse der Menschen passgenau aufnehmen sollen. Es gilt darum Versorgungsstrukturen aufzubauen, die Angehörige, Bekannte und Nachbarn einbeziehen und auch preisgünstige Dienste aus anderen europäischen Ländern.

\section{- Angebote der Diakonie in anderen europäi-} schen Ländern: Genauso wie diakonische Träger ihre Leistung über Grenzen der Kommunen und Bundesländer hinweg anbieten, können sie das auch über nationale Grenzen hinweg tun. Teilweise geschieht das schon. Denn für eine ausschließliche Antwort auf Bedürfnisse von Menschen in einem engen geographischen und politischen Raum gab es weder zu Wicherns Zeiten noch heute vernünftige oder gar diakonische Gründe. Mit unserem Menschenbild und unserer Fachlichkeit sind wir in anderen europäischen Ländern nicht nur für die dort lebenden Ausländer vertrauenswürdige und geschätzte Dienstleistungsanbieter, sondern auch für die dort lebende Bevölkerung. In einigen Ländern sind unsere Erfahrungen beim Aufbau entsprechender Versorgungsstrukturen ausgesprochen begehrt. Es bietet sich an, für solche Angebote transnationale Allianzen zu bilden.

- Veränderung unserer Vergütungssysteme: Wenn wir uns auf einen europäischen Markt einstellen wollen, dann müssen wir unsere Vergütungssysteme verändern. Angesichts dessen, dass unsere Kosten zu 70 Prozent von den Personalkosten bestimmt sind, sind unsere Angebote in einem europäischen Wettbewerb nur schwer konkurrenzfähig. Auf Dauer können wir bei den derzeitigen Personalkosten nur Angebote für ein oberes Preissegment und für den spendenfinanzierten Bereich machen.

\section{Perspektiven}

Diakonische Träger werden Europa als Verantwortungsraum für ihr Handeln begreifen lernen. Sie werden unabhängig von kommunalen und nationalen Grenzen bei den Menschen sein, die ihre Hilfe brauchen. Es werden sich transnationale Netzwerke im Bereich der Altenarbeit bilden, um vernetzte diakonische Angebote zu machen, um voneinander zu lernen und um passgenaue individuelle Angebote für die Menschen zu machen, denen unsere Arbeit gilt.

Auf diesem Wege können wir helfen, die diakonischen Angebotsstrukturen besonders in den süd- und osteuropäischen Ländern zu entwickeln, aufzubauen und zu stärken. Dazu haben sich europäische Arbeitsgemeinschaften und Netze gebildet, die ihre Arbeit abstimmen, Informationen und Mitarbeiter austauschen.

Eine im europäischen Horizont handelnde Diakonie kann die Besonderheit diakonischer Dienstleistungen mit ihrer ethischen und spirituellen Dimension und Verpflichtung in die europäische Entwicklung einbringen und darauf achten, dass die europäische Rechtsentwicklung die Handlungsspielräume für diakonisches Handeln nicht einschränkt.

Diakonie ist nicht nur von Europa betroffen, sondern sie kann auch Europa mitgestalten. Zunächst ist das die Möglichkeit vor allem für den Spitzenverband, dann aber auch für die diakonischen Träger. Diakonie erhält damit die Chance, den nationale Grenzen überschreitenden diakonischen Auftrag wahrzunehmen. Impulse dafür sind seit Wichern in der Diakonie wirksam.

Die Realität zeigt: Die Anzahl der Träger, die sich mittlerweile in Europa engagieren, ist deutlich gestiegen, wobei das Engagement vielschichtig ist. Die verschiedenen Formen von Beratung, Unterstützung, Wissenstransfer bis hin zu realisierten Projekten mittels eigener Dependancen im jeweiligen Land zeigen, dass Diakonie sich bereits auf den Weg gemacht hat.

\section{Resümee}

Die Schlüsselfrage für europäische Sozialdienste ist: wie Pflege bei guter Qualität bezahlbar bleibt und gestaltet werden kann. Höhere Lebenserwartung und zunehmende Pflegebedürftigkeit, sinkende Geburtenraten und Belastbarkeitsgrenzen in der Familie machen grenzüberschreitende Anstrengungen erforderlich. Es gilt Versorgungsstrukturen aufzubauen, die sowohl Bezugspersonen als auch preisgünstige Dienste aus anderen europäischen Ländern einbeziehen. Vergütungssysteme sind zu verändern, um konkurrenzfähig zu bleiben. Schließlich sind diakonische Netzwerke zu bilden, die ihre Arbeit abstimmen und Erfahrungen austauschen.

\section{Anmerkungen}

(1) Brian Munday, Europäische Sozialdienste. Eine Übersicht der Merkmale und Trends, Kent University 2003.

(2) Daten aus Eurostat, Statistik kurzgefasst, Thema 3, 1/2004.

(3) Brian Munday, ebd. 